\title{
Introduction to High Energy Physics
}

4th Edition

Donald H. Perkins

University of Oxford 


\section{Contents}

1 Quarks and leptons 1

1.1 Preamble 1

1.2 The Standard Model of particle physics 7

$\begin{array}{lr}1.3 \text { Particle classification: fermions and bosons } & 12\end{array}$

$\begin{array}{ll}1.4 & \text { Particles and antiparticles } \\ 13\end{array}$

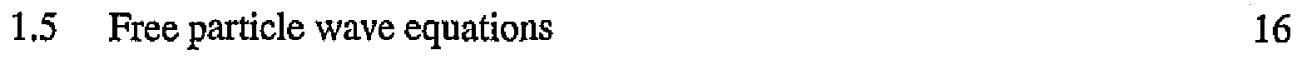

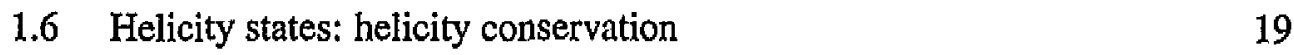

$\begin{array}{ll}1.7 \text { Lepton flavours } & 20\end{array}$

$\begin{array}{lll}1.8 \text { Quark flavours } & 22\end{array}$

1.9 The cosmic connection $\quad 26$

$\begin{array}{ll}\text { Problems } & 33\end{array}$

$2 \quad$ Interactions and fields $\quad \mathbf{3 5}$

2.1 Classical and quantum pictures of interactions 35

2.2 The Yukawa theory of quantum exchange 36

2.3 The boson propagator $\quad 37$

2.4 Feynman diagrams $\quad 38$

2.5 Electromagnetic interactions $\quad 40$

2.6 Renormalisation and gauge invariance $\quad 42$

2.7 Strong interactions $\quad 43$

2.8 Weak and electroweak interactions 46

2.9 Gravitational interactions 51

2.10 The interaction cross-section $\quad 51$

2.11 Decays and resonances $\quad 55$

Problems $\quad 61$ 
3 Invariance principles and conservation laws 63

3.1 Translation and rotation operators 63

3.2 The parity operation 65

3.3 Pion spin and parity 66

3.4 Parity of particles and antiparticles 69

$\begin{array}{lll}3.5 & \text { Tests of parity conservation } & 72\end{array}$

3.6 Charge conjugation invariance 73

3.7 Charge conservation and gauge invariance 75

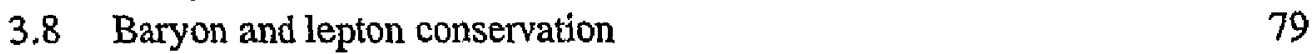

$3.9 C P T$ invariance $\quad 81$

$3.10 C P$ violation and $T$ violation $\quad 81$

3.11 Neutron electric dipole moment 83

3.12 Isospin symmetry 87

3.13 Isospin in the two-nucleon and pion-nucleon systems 88

3.14 Isospin, strangeness and hypercharge 91

Problems $\quad 93$

4 Quarks in hadrons 95

4.1 Charm and beauty; the heavy quarkonium states 95

4.2 Comparison of quarkonium and positronium levels 102

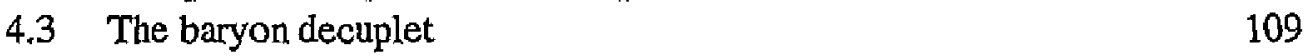

4.4 Quark spin and colour $\quad 114$

4.5 The baryon octet 115

4.6 Quark-antiquark combinations: the light pseudoscalar mesons 118

4.7 The light vector mesons 121

4.8 Other tests of the quark model 123

4.9 Mass relations and hyperfine interactions 126

4.10 Electromagnetic mass differences and isospin symmetry 129

4.11 Magnetic moments of baryons 130

4.12 Mesons built of light and heavy quarks 132

4.13 The top quark 134

$\begin{array}{ll}\text { Problems } & 139\end{array}$

$5 \quad$ Lepton and quark scattering $\quad 140$

5.1 The process $e^{+} e^{-} \rightarrow \mu^{+} \mu^{-}-140$

$5.2 e^{+} e^{-}$annihilation to hadrons 144

5.3 Electron-muon scattering, $e^{-} \mu^{+} \rightarrow e^{-} \mu^{+} \quad 147$

5.4 Neutrino-electron scattering, $v_{e} e \rightarrow v_{e} e \quad 150$

5.5 Elastic lepton-nucleon scattering 154

5.6 Deep inelastic scattering and partons 155

5.7 Deep inelastic scattering and quarks $\quad 159$ 
5.8 Experimental results on quark distributions in the nucleon 162

5.9 Sum rules 166

5.10 Summary 168

$\begin{array}{ll}\text { Problems } & 168\end{array}$

6 Quark interactions and QCD 171

6.1 The colour quantum number 171

6.2 The QCD potential at short distances 172

6.3 The QCD potential at large distances: the string model 178

6.4 Gluon jets in $e^{+} e^{-}$annihilation 180

6.5 Running couplings in QED and QCD 181

6.6 Evolution of structure functions in deep inelastic scattering 186

6.7 Gluonium and the quark-gluon plasma 190 $\begin{array}{ll}\text { Problems } & 192\end{array}$

7 Weak interactions 194

7.1 Classification 194

7.2 Lepton universality 195

7.3 Nuclear $\beta$-decay: Fermi theory 197

7.4 Inverse $\beta$-decay: neutrino interactions 201

7.5 Parity nonconservation in $\beta$-decay 202

7.6 Helicity of the neutrino 205

7.7 The $V-A$ interaction 206

7.8 Conservation of weak currents 209

7.9 The weak boson and Fermi couplings 210

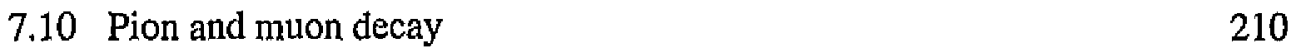

7.11 Neutral weak currents 213

7.12 Observation of $W^{ \pm}$and $Z^{0}$ bosons in $p \bar{p}$ collisions 215

$\begin{array}{ll}7.13 Z^{0} \text { production at } e^{+} e^{-} \text {colliders } & 220\end{array}$

7.14 Weak decays of quarks. The GIM model and the CKM matrix 221

7.15 Neutral $K$ mesons $\quad 226$

$7.16 C P$ violation in the neutral kaon system 232

$\begin{array}{ll}7.17 \text { Cosmological } C P \text { violation } & 237\end{array}$

$7.18 D^{0}-\bar{D}^{0}$ and $B^{0}-\bar{B}^{0}$ mixing $\quad 238$

Problems 239

8 Electroweak interactions and the Standard Model 242

8.1 Introduction 242

8.2 Divergences in the weak interactions 243

8.3 Introduction of neutral currents 245

8.4 The Weinberg-Salam model 246 
8.5 Intermediate boson masses 248

8.6 Electroweak couplings of leptons and quarks 249

8.7 Neutrino scattering via $Z$ exchange 250

8.8 Asymmetries in the scattering of polarised electrons by deuterons 253

8.9 Observations on the $Z$ resonance 255

8.10 Fits to the Standard Model and radiative corrections 260

$\begin{array}{ll}8.11 W \text { pair production } & 262\end{array}$

8.12 Spontaneous symmetry breaking and the Higgs mechanism 263

$\begin{array}{ll}8.13 \text { Higgs production and detection } & 271\end{array}$ $\begin{array}{ll}\text { Problems } & 274\end{array}$

9 Physics beyond the Standard Model 276

$\begin{array}{lll}9.1 & \text { Supersymmetry } & 277\end{array}$

9.2 Grand unified theories: the SU(5) GUT 278

9.3 Unification energy and weak mixing angle 280

9.4 Supersymmetric SU(5) 282

9.5 Proton decay 282

9.6 Neutrino mass: Dirac and Majorana neutrinos 284

9.7 Neutrino oscillations $\quad 287$

9.8 Magnetic monopoles $\quad 299$

9.9 Superstrings 300

Problems 301

10 Particle physics and cosmology 303

10.1 Hubble's law and the expanding universe 303

10.2 Friedmann equation 304

10.3 Cosmic microwave radiation: the hot Big Bang 307

10.4 Radiation and matter eras 311

10.5 Nucleosynthesis in the Big Bang 313

$\begin{array}{ll}10.6 \text { Baryon-antibaryon asymmetry } & 317\end{array}$

$\begin{array}{ll}10.7 \text { Dark matter } & 319\end{array}$

10.8 Inflation $\quad 326$

10.9 Neutrino astronomy: SN 1987A $\quad 330$

Problems $\quad 336$

11 Experimental methods $\quad 338$

11.1 Accelerators 338

11.2 Colliding-beam accelerators $\quad 343$

11.3 Accelerator complexes $\quad 346$

11.4 Secondary particle separators $\quad 346$

11.5 Interaction of charged particles and radiation with matter 349 
11.6 Detectors of single charged particles 355

11.7 Shower detectors and calorimeters 368

$\begin{array}{ll}\text { Problems } & 375\end{array}$

Appendix A Table of elementary particles $\quad 377$

$\begin{array}{lll}\text { Appendix } B & \text { Milestones in particle physics } & 379\end{array}$

Appendix C Clebsch-Gordan coefficients and d-functions 386

Appendix D Spherical harmonics, $d$-functions and Clebsch-Gordan coefficients 393

Appendix $E$ Relativistic normalisation of cross-sections and decay rates 396

$\begin{array}{ll}\text { Glossary } & 398\end{array}$

Answers to problems $\quad 408$

\begin{tabular}{lr} 
Bibliography & 412 \\
\hline Refences & 418
\end{tabular}

References 418

$\begin{array}{ll}\text { Index } & 421\end{array}$ 